\title{
REAVALIAÇÃO DE CIRCUITO DE FLOTAÇÃO CONVENCIONAL DE MINÉRIO DE FERRO*
}

\author{
Airton Antônio Silva ${ }^{1}$ \\ Antônio Eduardo Clark Peres ${ }^{2}$ \\ Paulo Sérgio de Oliveira ${ }^{3}$ \\ João Paulo Melo Silva ${ }^{4}$
}

\section{Resumo}

A Samarco Mineração, desde o início de suas atividades em 1977 na mina de Germano, tem sido pioneira na lavra e beneficiamento de minério de ferro friável e de baixo teor (itabiritos). Atualmente, a queda no teor de Fe associada à maior participação de materiais compactos, semi-compactos e com maior presença de contaminantes tem impactado na recuperação do circuito de flotação convencional do concentrador I. A redução da recuperação nessa etapa de flotação motivou a reavaliação do circuito de flotação atual buscando alternativas para reestabelecer a recuperação sem afetar o teor de concentrado final e com menor investimento. Foram realizadas amostragens, caracterização física e química das amostras e, em seguida, execução dos ensaios de flotação em bancada, balanço de massas e desenvolvimento de modelos para a simulação de circuitos. Os resultados analisados mostraram oportunidades de melhoria do desempenho do circuito. O circuito de flotação antes da modificação era composto das etapas: rougher, cleaner, recleaner e scavenger. Com a reavaliação do circuito, a recleaner foi transformada em cleaner da scavenger. O concentrado scavenger antes da modificação possuía teores de $23 \% \mathrm{Fe}$ e representava $38 \%$ da alimentação nova. Com a modificação do circuito, a carga circulante foi reduzida de $36 \%$ para $15 \%$ e o teor de ferro subiu para $41,7 \%$, aumentando o tempo de residência. O teor de ferro no rejeito global foi reduzido de $10,92 \%$ (média 2013) para 10,2\% gerando ganhos de recuperação em massa e metalúrgica.

Palavras-chave: Itabiritos pobres; Flotação; Circuitos; Carga circulante.

\section{REEVALUATION OF IRON ORE CONVENCIONAL FLOTATION CIRCUIT Abstract}

Samarco Mineração, since the start up in 1977 at Germano's mine, has been a pioneer in mining and beneficiation of low grade friable iron ore (itabirite). Currently the decrease in iron content and increase in contaminants grade, associated with greater participation of compact and semi-compact materials, has impacted the recovery of concentrator I conventional flotation circuit. The recovery reduction in the flotation stage motivated the current flotation circuit reevaluation seeking alternatives to restore the recovery without affecting the final concentrate content with low investment. Sampling, physical and chemical characterization of samples, bench scale flotation tests, mass balance and development of models for circuit simulation were conducted. Analysis of the results showed opportunities to improve the circuit performance. The stages prior to the flotation circuit change were: rougher, cleaner, recleaner and scavenger. After the circuit reevaluation, the recleaner was transformed into cleaner scavenger. Prior to the modification the iron grade in the scavenger concentrate was $23 \%$, representing $38 \%$ of the new feed. With the circuit modification, the circulating load was reduced from $36 \%$ to $15 \%$ and the iron content increased to $41,7 \%$, resulting in increased residence time. The iron content in the global talings was decreased from $10.92 \%$ ( 2013 average) to $10.2 \%$ representing gains in both mass and metallurgical recoveries.

Keywords: Low grade itabirites; Flotation circuits; Circulating load.

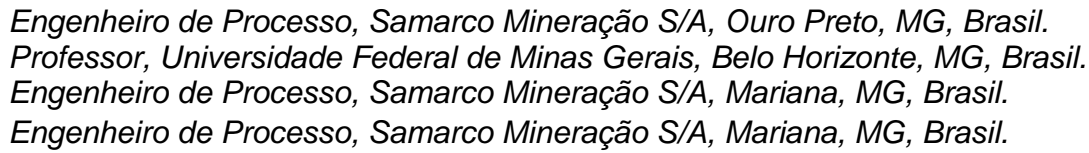




\section{INTRODUÇÃO}

A flotação é um dos principais processos de concentração, sendo empregado para diversas classes de minerais (sulfetos, óxidos, silicatos, fosfatos, etc.), como também para uma variedade de tamanhos de partículas. Estudos anteriores mostraram que a flotação pode ser empregada com sucesso para faixas granulométricas entre 10 e $300 \mu \mathrm{m}$. A seletividade do processo de flotação torna-se muito baixa fora desses limites granulométricos porque as condições hidrodinâmicas do sistema são incapazes de manter o nível de flotabilidade ideal das partículas [1].

A flotação é uma técnica que faz uso das diferenças de características físico-químicas de superficie dos minerais para separá-los. Apesar de sua complexidade, a flotação é uma técnica versátil e eficiente para recuperação de espécies minerais que não poderiam ser concentradas por outras técnicas. As variáveis operacionais da flotação são ajustadas com base nas características do minério. Parâmetros como: reagentes (coletores, depressores e modificadores), granulometria, $\mathrm{pH}$, percentual de sólidos na polpa, tempo de condicionamento, temperatura, entre outros, devem ser cuidadosamente ajustados visando uma maior eficiência do processo de flotação.

Para a seleção de fluxogramas de processamento de minérios de ferro existem alguns fatores importantes a serem considerados e esses podem ser divididos em fatores intrínsecos e extrínsecos ao minério que se deseja processar. Como fatores intrínsecos deve-se considerar: as características físico-químicas do ROM (run of mine), a distribuição granulométrica, o grau de liberação das partículas minerais, os teores químicos e as associações mineralógicas do minério. Como fatores extrínsecos, pode-se considerar: as características físico-químicas desejadas para o seu transporte e destinação nas etapas metalúrgicas subsequentes, como por exemplo, aglomeração (briquetagem, sinterização e pelotização), redução em altoforno ou processos de redução direta; tais características ditarão os quesitos de qualidades físicas, químicas e metalúrgicas para esses produtos. Adicionalmente, os fatores econômicos envolvidos nas operações de adequação dessa matéria-prima serão avaliados na definição das rotas de processo [1].

Os minérios com elevados teores de Fe (minérios ricos), que atendem naturalmente às especificações químicas do mercado consumidor, são tipicamente processados em circuitos de fragmentação, classificação por tamanho (processo a úmido) e desaguamento. As operações de classificação a úmido e desaguamento favorecem a remoção das lamas argilosas, onde se concentram as impurezas indesejáveis, principalmente fósforo e alumina [2,3].

Para a concentração de minérios de baixos teores de ferro (minérios pobres) são utilizados, em geral, os métodos gravíticos, de concentração magnética e flotação. Segundo Viana [4], são inúmeras as possibilidades de combinação de processos unitários e tipos de equipamentos para o beneficiamento de minérios em diferentes sistemas minerais, o que pode levar à suposição de que para um tipo específico de minério existirá uma solução única. Embora a natureza de sistemas muito diferenciados, como por exemplo, minérios de ferro itabiríticos e minérios sulfetados de cobre, requereria a adoção de algumas práticas e tipos de circuitos característicos para cada um desses dois sistemas, a experiência industrial tem demonstrado que muitos minérios, eventualmente considerados como de um tipo único, mostram variações significativas nas suas características físicas, químicas e mineralógicas que levam a divisões do mesmo em dezenas de subtipos.

Para a seleção do fluxograma mais adequado, deve-se sempre considerar a combinação de métodos de beneficiamento que possam fornecer a flexibilidade 
adequada às variações nas características do minério considerado como único, estando a variabilidade associada às diferentes espécies mineralógicas e suas propriedades intrínsecas. Outro aspecto a ser considerado, ao se tratar de fluxogramas, é a interdependência dos diversos processos unitários existentes no circuito industrial. A modificação nas variáveis de processo ou de projeto de um determinado processo pode gerar consequências importantes nos processos subsequentes localizados em outra parte do circuito, limitando ou melhorando o desempenho desse segundo processo. Assim, a condição ótima de operação de um processo específico dentro de um fluxograma, deve ser vista de uma maneira integrada em todo o fluxograma. Minérios itabiríticos, que requerem moagem fina para a liberação dos minerais de ganga, são concentrados, na maioria dos casos, por separação magnética de alta intensidade a úmido e flotação catiônica reversa, sendo comuns as combinações desses processos. Exemplos clássicos de aplicação de separação magnética de alta intensidade são as usinas de Cauê e Conceição (VALE) [5].

Segundo Viana [4], apesar da combinação de diferentes métodos ser cada vez mais empregada, a flotação vem ampliando aceleradamente sua participação como método de concentração, devido principalmente à sua comprovada seletividade para minérios de ferro e à ampla faixa de tamanho e teores de ROM em que ela se aplica.

\section{MATERIAL E MÉTODOS}

Em 19/09/2013 foi realizada amostragem geral do concentrador I e o balanço de massa mostrou oportunidade de reavaliar o circuito de flotação convencional, pois o desempenho dos estágios cleaner e recleaner estava comprometendo o desempenho do circuito da flotação. Campanhas de amostragens foram realizadas neste circuito para confirmar os dados. Foram realizadas 16 amostragens e ficou evidente que em alguns momentos a etapa recleaner não era demandada. Notou-se ainda que a carga circulante apresentava teores de ferro próximos de $23 \%$ e esse material médio se juntava à alimentação nova que possui teores de aproximadamente $44 \%$ de ferro empobrecendo a mesma.

A metodologia utilizada neste estudo incluiu as seguintes etapas:

- coleta e preparação das amostras;

- caracterização física e química das amostras;

- execução dos ensaios de flotação em bancada.

Foi coletada uma amostra de minério de ferro na alimentação da etapa de flotação convencional do concentrador 1 , no dia 22/01/2014 durante $2 \mathrm{~h}$ com incrementos a cada 15 minutos. Com esta amostra foram realizados dois testes de flotação em bancada conforme procedimento abaixo:

Testes 01:

- Flotar alimentação durante 1,5 min e retirar concentrado e rejeito rougher (10 testes para acumular massa);

- Flotar concentrado rougher durante 1,5 min e retirar concentrado e rejeito cleaner (10 testes);

- Flotar concentrado cleaner durante 1,5 min e retirar concentrado e rejeito recleaner (01 teste);

- Juntar os rejeitos rougher e cleaner dos testes anteriores e flotar (03 testes para gerar concentrados e rejeito final);

- Nos testes de flotação scavenger ajustar os percentuais de sólidos da alimentação para $40 \%$; 
- Adicionar amido na alimentação das scavengers da rougher e cleaner até ajustar pH para 10,5.

- Retirar uma alíquota da alimentação, concentrado e rejeito de cada etapa para Teste 02: análise química.

- Flotar alimentação durante 1,5 min e retirar concentrado e rejeito rougher (10 testes para acumular massa);

- Flotar concentrado rougher durante 1,5 min e retirar concentrado e rejeito cleaner (10 testes);

- Juntar os rejeitos rougher e cleaner dos testes anteriores e flotar (10 testes para gerar concentrados que irão alimentar a flotação cleaner da scavenger e rejeito final);

- Flotar os concentrados cleaner da scavenger (01 teste concentrado e rejeito final);

- Nos testes de flotação scavenger ajustar os percentuais de sólidos da alimentação para $40 \%$;

- Adicionar amido na alimentação das scavengers da rougher e cleaner até ajustar o pH para 10,5.

- Retirar uma alíquota da alimentação, concentrado e rejeito de cada etapa para análise química.

Os testes foram realizados em uma célula de flotação WEMCO de $2.400 \mathrm{~mL}$, com rotação de 1.300rpm. O depressor dos minerais de ferro utilizado nos dois testes foi o amido de mandioca gelatinizado com hidróxido de sódio, na proporção mássica 5:1. O coletor empregado nos testes foi uma eteramina. As soluções de depressor, hidróxido de sódio e coletor foram preparadas nas seguintes concentrações: $1 \% \mathrm{p} / \mathrm{v}$, $3 \% \mathrm{p} / \mathrm{v}$ e $1 \% \mathrm{p} / \mathrm{v}$, respectivamente. A massa de sólido utilizada nos ensaios de flotação foi de $1,5 \mathrm{~kg}$ e o percentual de sólido (p/p) 42,0\%. O depressor foi condicionado durante cinco minutos e o coletor condicionado durante um minuto. A análise química foi realizada por dicromatometria, por análise em espectrômetro de emissão ótica com plasma acoplado e por gravimetria.

Com os resultados foram realizados balanços de massas e simulações do circuito de flotação convencional atual e do circuito de flotação proposto.

\section{RESULTADOS E DISCUSSÃO}

O circuito de flotação convencional era composto pelas etapas rougher, cleaner, recleaner e scavenger conforme mostrado na figura 1.

Os resultados do balanço de massa referente às amostragens industriais e o balanço de massas realizado com os testes de bancada estão apresentados a seguir nas tabelas 1 e 2 respectivamente. 


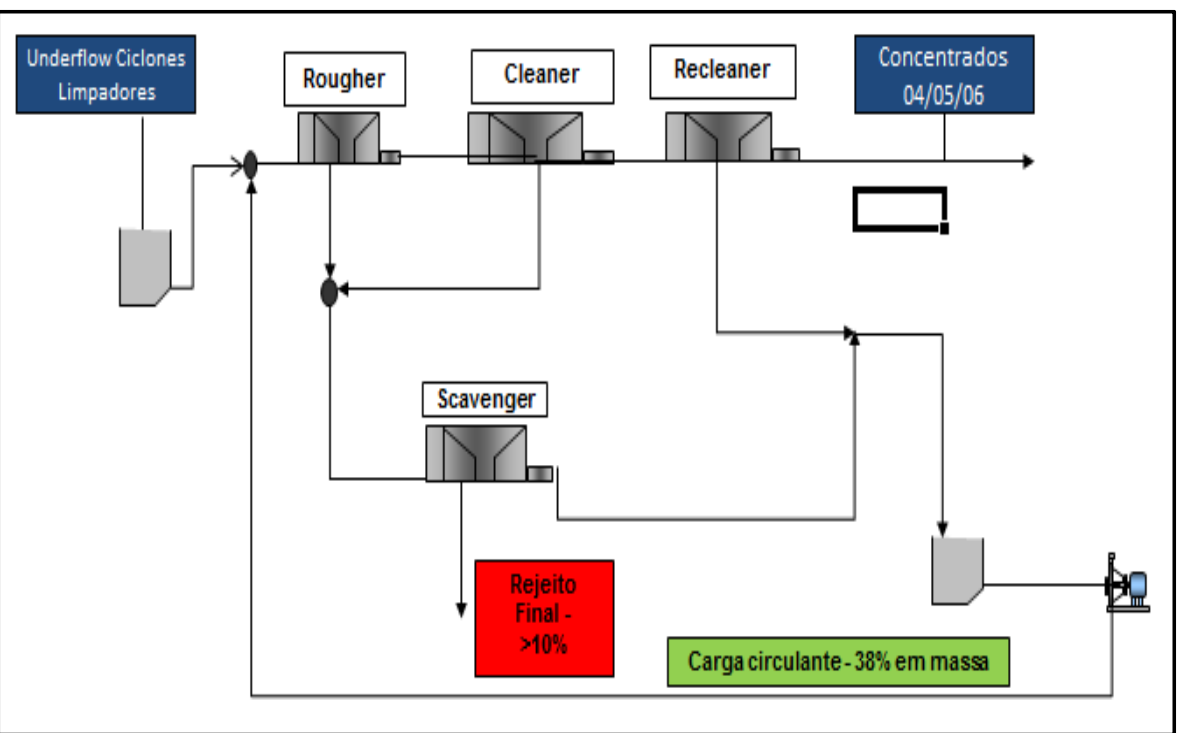

Figura 1: Circuito da flotação convencional antes da modificação.

A tabela 1 apresenta os resultados da amostragem industrial antes da modificação do circuito. Nota-se que a recuperação em peso da etapa cleaner foi de $88,45 \%$, o teor de ferro no rejeito global foi de $8,98 \%$ e o teor de $\mathrm{SiO}_{2}$ no concentrado cleaner foi de $2,21 \%$. Apesar do teor de ferro no rejeito apresentar-se baixo em alguns momentos, o concentrado scavenger também possui teor de Fe próximo a $26 \%$, sendo esse incompatível com o teor de Fe da alimentação nova.

Tabela 1 - Resultados do balanço de massa de uma das amostragens industriais do circuito de flotação convencional antes da modificação.

\begin{tabular}{|c|c|c|c|c|c|}
\hline \multicolumn{2}{|c|}{ Etapa de flotação } & \multirow{2}{*}{$\begin{array}{l}\% \mathrm{Fe} \\
42,89 \\
\end{array}$} & \multirow{2}{*}{$\begin{array}{c}\% \text { Silica } \\
37,43 \\
\end{array}$} & \multirow{4}{*}{\begin{tabular}{|c|} 
Rec.Peso \\
62,5 \\
\end{tabular}} & \multirow{4}{*}{\begin{tabular}{|c} 
Rec Metálica \\
87,84
\end{tabular}} \\
\hline Alimentação & Rougher & & & & \\
\hline Concentado & Rougher & 60,28 & 10,21 & & \\
\hline Rejeito & Rougher & 13,9 & 79,67 & & \\
\hline Alimentação & Cleaner & 60,28 & 10,21 & \multirow{3}{*}{88,45} & \multirow{3}{*}{96,14} \\
\hline Concentado & Cleaner & 65,52 & 3,97 & & \\
\hline Rejeito & Cleaner & 20,15 & 70,44 & & \\
\hline Alimentação & Recleaner & 65,52 & 3,97 & \multirow{3}{*}{94,15} & \multirow{3}{*}{96,23} \\
\hline Concentado & Recleaner & 66,97 & 2,21 & & \\
\hline Rejeito & Recleaner & 42,16 & 37,55 & & \\
\hline Alimentação & Scavenger & 14,2 & 79,21 & \multirow{3}{*}{30,0} & \multirow{3}{*}{55,71} \\
\hline Concentado & Scavenger & 26,37 & 64,99 & & \\
\hline Rejeito & Scavenger & 8,98 & 86,80 & & \\
\hline \multicolumn{2}{|c|}{ Rejeito e recuperação global } & 8,98 & & 58,47 & 91,3 \\
\hline
\end{tabular}

$\mathrm{Na}$ tabela 2 os resultados do balanço de massas do teste de bancada para o circuito de flotação convencional antes da modificação mostram recuperação em peso global de $66 \%$, recuperação metálica de $92 \%$. O teor de ferro no rejeito final foi de $10,92 \%$, a sílica no concentrado recleaner foi de $4,48 \%$ e o teor de ferro da carga circulante foi de $24 \%$, o que confirma os dados das amostragens industriais. 
Tabela 2 - Resultados dos testes de bancada para o circuito de flotação convencional antes da modificação.

\begin{tabular}{|c|c|c|c|c|c|c|}
\hline \multicolumn{7}{|c|}{ Tabela de resultados antes da modificação do circuito } \\
\hline \multicolumn{2}{|c|}{ Etapa de flotação } & $\% \mathrm{Fe}$ & \%Silica & Rec.Peso & Rec Metálica & $\begin{array}{c}\text { Indice de } \\
\text { seletividade global }\end{array}$ \\
\hline \multicolumn{2}{|c|}{ Alimentação Nova } & 46,45 & & & & \\
\hline Alimentação & Rougher & 44,33 & 33,64 & \multirow{3}{*}{0,615} & \multirow{3}{*}{0,879} & \multirow{12}{*}{10,11} \\
\hline Concentado & Rougher & 63,42 & 6,35 & & & \\
\hline Rejeito & Rougher & 13,9 & 70,36 & & & \\
\hline Alimentação & Cleaner & 63,42 & 6,35 & \multirow{3}{*}{0,987} & \multirow{3}{*}{0,996} & \\
\hline Concentado & Cleaner & 64,00 & 5,38 & & & \\
\hline Rejeito & Cleaner & 20,15 & 62,25 & & & \\
\hline Alimentação & Recleaner & 64,00 & 5,38 & \multirow{3}{*}{0,968} & \multirow{3}{*}{0,978} & \\
\hline Concentado & Recleaner & 64,72 & 4,48 & & & \\
\hline Rejeito & Recleaner & 42,16 & 33,71 & & & \\
\hline Alimentação & Scavenger & 14,03 & 70,19 & \multirow{3}{*}{0,233} & \multirow{3}{*}{0,403} & \\
\hline Concentado & Scavenger & 24,26 & 56,92 & & & \\
\hline Rejeito & Scavenger & 10,92 & 74,23 & & & \\
\hline \multicolumn{2}{|c|}{ Rejeito e recuperações global } & 10,92 & & 0,660 & 0,920 & \\
\hline
\end{tabular}

O fluxograma do circuito proposto está mostrado na figura 2 e os dados do balanço de massas, a partir dos testes de bancada, estão mostrados na tabela 3.

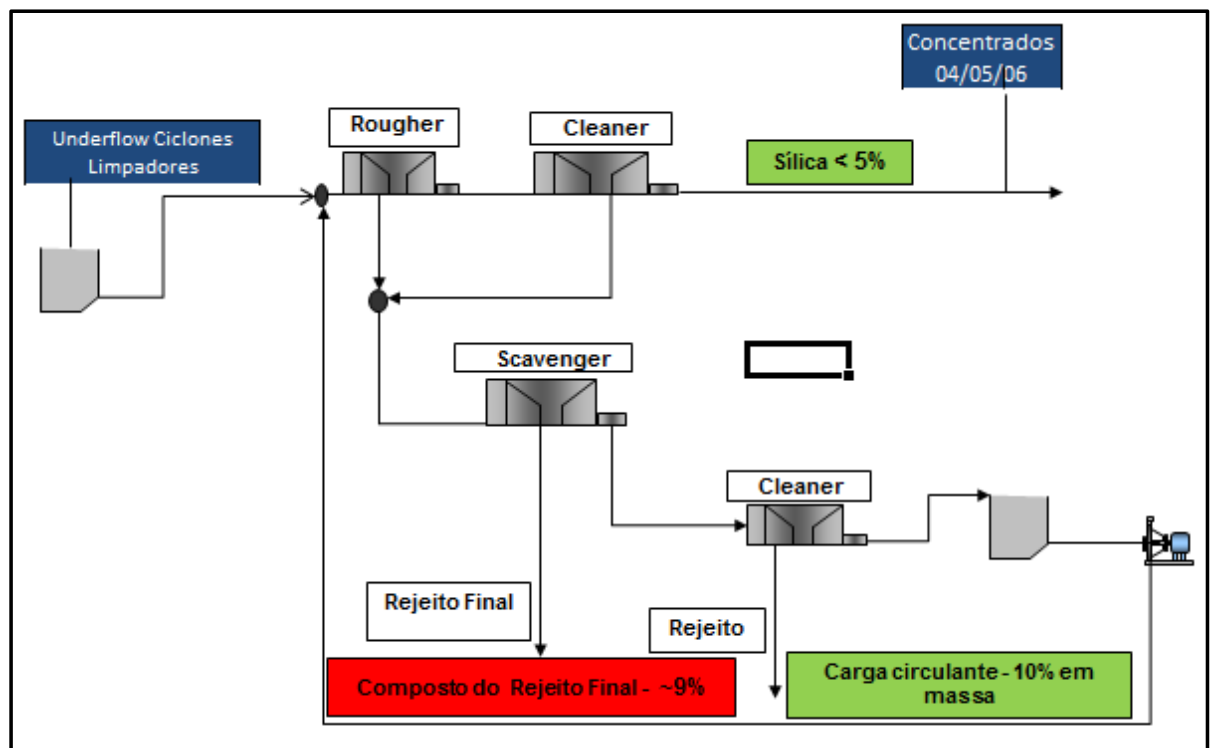

Figura 2: Circuito da flotação convencional após a modificação.

De acordo com os resultados do teste de bancada para o circuito proposto mostrado na tabela 3, nota-se que o teor de ferro no rejeito global foi de $9,67 \%$ e o teor de $\mathrm{SiO}_{2}$ no concentrado cleaner foi de $4,44 \%$. A recuperação em massa foi de $66,8 \%$ e a metálica de 93,0\%. A carga circulante apresentou teor próximo da alimentação nova (39\%), o que motivou a modificação em uma das linhas de flotação. 
Tabela 3 - Resultados dos testes de bancada para o circuito de flotação convencional após a modificação do circuito.

\begin{tabular}{|c|c|c|c|c|c|c|}
\hline \multicolumn{7}{|c|}{ Tabela de resultados do circuito modificado } \\
\hline \multicolumn{2}{|c|}{ Etapa de flotação } & $\% \mathrm{Fe}$ & $\%$ Silica & Rec.Peso & Rec Metálica & \begin{tabular}{|c|} 
Indice de \\
seletividade global
\end{tabular} \\
\hline \multicolumn{2}{|c|}{ Alimentação Nova } & 46,45 & & & & \\
\hline Alimentação & Rougher & 45,8 & 31,54 & \multirow{3}{*}{64,16} & \multirow{3}{*}{88,25} & \multirow{12}{*}{10,86} \\
\hline Concentado & Rougher & 63,00 & 6,95 & & & \\
\hline Rejeito & Rougher & 15,00 & 68,93 & & & \\
\hline Alimentação & Cleaner & 63,00 & 6,95 & \multirow{3}{*}{95,0} & \multirow{3}{*}{97,60} & \\
\hline Concentado & Cleaner & 64,72 & 4,44 & & & \\
\hline Rejeito & Cleaner & 30,00 & 49,48 & & & \\
\hline Alimentação & Scavenger & 16,00 & 74,13 & \multirow{3}{*}{53,33} & \multirow{3}{*}{76,62} & \\
\hline Concentado & Scavenger & 23,00 & 58,56 & & & \\
\hline Rejeito & Scavenger & 8,00 & 78,01 & & & \\
\hline Alimentação & Cleaner da Scavenger & 23,00 & 64,12 & \multirow{3}{*}{40,70} & \multirow{3}{*}{69,01} & \\
\hline Concentado & Cleaner da Scavenger & 39,00 & 37,81 & & & \\
\hline Rejeito & Cleaner da Scavenger & 12,00 & 72,83 & & & \\
\hline \multicolumn{2}{|c|}{ Rejeito e recuperação global } & 9,67 & & 0,668 & 0,930 & \\
\hline
\end{tabular}

De posse das amostragens e dos resultados em bancada, decidiu-se por modificar uma das linhas de flotação. A proposta foi transformar a célula de flotação recleaner em cleaner da scavenger devido a configuração dos bancos no campo.

Após modificação do circuito da linha 01 , foi realizada amostragem industrial no dia 25/02/14 e os dados estão apresentados na tabela 04.

Os resultados do teste foram muito promissores: o teor de ferro no rejeito na etapa scavenger foi de $8,5 \%$, na etapa cleaner da scavenger $14,8 \%$ compondo um rejeito global de $11,8 \%$; o teor de ferro da carga circulante foi de $41,7 \%$, que é um teor compatível com a alimentação nova do circuito e sua massa foi reduzida de $36 \%$ para $15 \%$ durante os testes na linha 01 . Diante dos resultados obtidos partiu-se para modificação da linha 02 .

Tabela 4 - Resultados dos testes industriais realizados na linha 01 após a modificação do circuito.

\begin{tabular}{|c|c|c|c|c|c|c|}
\hline \multicolumn{7}{|c|}{ Tabela de resultados do teste industrial- linha 01 para o circuito modificado } \\
\hline \multicolumn{2}{|c|}{ Etapa de flotação } & $\% \mathrm{Fe}$ & \%Silica & Rec.Peso & Rec Metálica & $\begin{array}{c}\text { Indice de } \\
\text { seletividade global }\end{array}$ \\
\hline \multicolumn{2}{|c|}{ Alimentação Nova } & 45,78 & & & & \\
\hline Alimentação & Rougher & 45,24 & 29,71 & \multirow{3}{*}{81,37} & \multirow{3}{*}{94,18} & \multirow{12}{*}{14,47} \\
\hline Concentado & Rougher & 52,36 & 20,47 & & & \\
\hline Rejeito & Rougher & 14,13 & 70,06 & & & \\
\hline Alimentação & Cleaner & 52,36 & 20,47 & \multirow{3}{*}{66,24} & \multirow{3}{*}{84,06} & \\
\hline Concentado & Cleaner & 66,45 & 2,20 & & & \\
\hline Rejeito & Cleaner & 24,71 & 23,65 & & & \\
\hline Alimentação & Scavenger & 20,43 & 61,89 & \multirow{3}{*}{66,20} & \multirow{3}{*}{85,94} & \\
\hline Concentado & Scavenger & 26,52 & 53,99 & & & \\
\hline Rejeito & Scavenger & 8,50 & 76,98 & & & \\
\hline Alimentação & Cleaner da Scavenger & 26,52 & 53,99 & \multirow{3}{*}{43,64} & \multirow{3}{*}{68,63} & \\
\hline Concentado & Cleaner da Scavenger & 41,71 & 34,29 & & & \\
\hline Rejeito & Cleaner da Scavenger & 14,76 & 69,25 & & & \\
\hline \multicolumn{2}{|c|}{ Rejeito e recuperação global } & 11,79 & & 62,18 & 90,30 & \\
\hline
\end{tabular}

Após modificação das linhas 01 e 02 , notou-se que o circuito dessas linhas demandava menor quantidade de amina em comparação com circuitos não modificados (linhas 03 e 04). As amostragens foram realizadas no período de 20/10/2014 a 25/11/2014. Foram coletados 850 dados horários das linhas 01 e 02 e 874 dados horários das linhas 03 e 04 . Os resultados estão mostrados na figura 03. A modificação do circuito foi estendida para as linhas 03 e 04, sendo concluída a modificação na linha 04 no dia 20/10/14. 
Ajustes na flotação são necessários para obter teores de ferro nos rejeitos globais menores que $10 \%$, pois, o teor de ferro no concentrado da cleaner da scavenger está compatível com a alimentação nova.

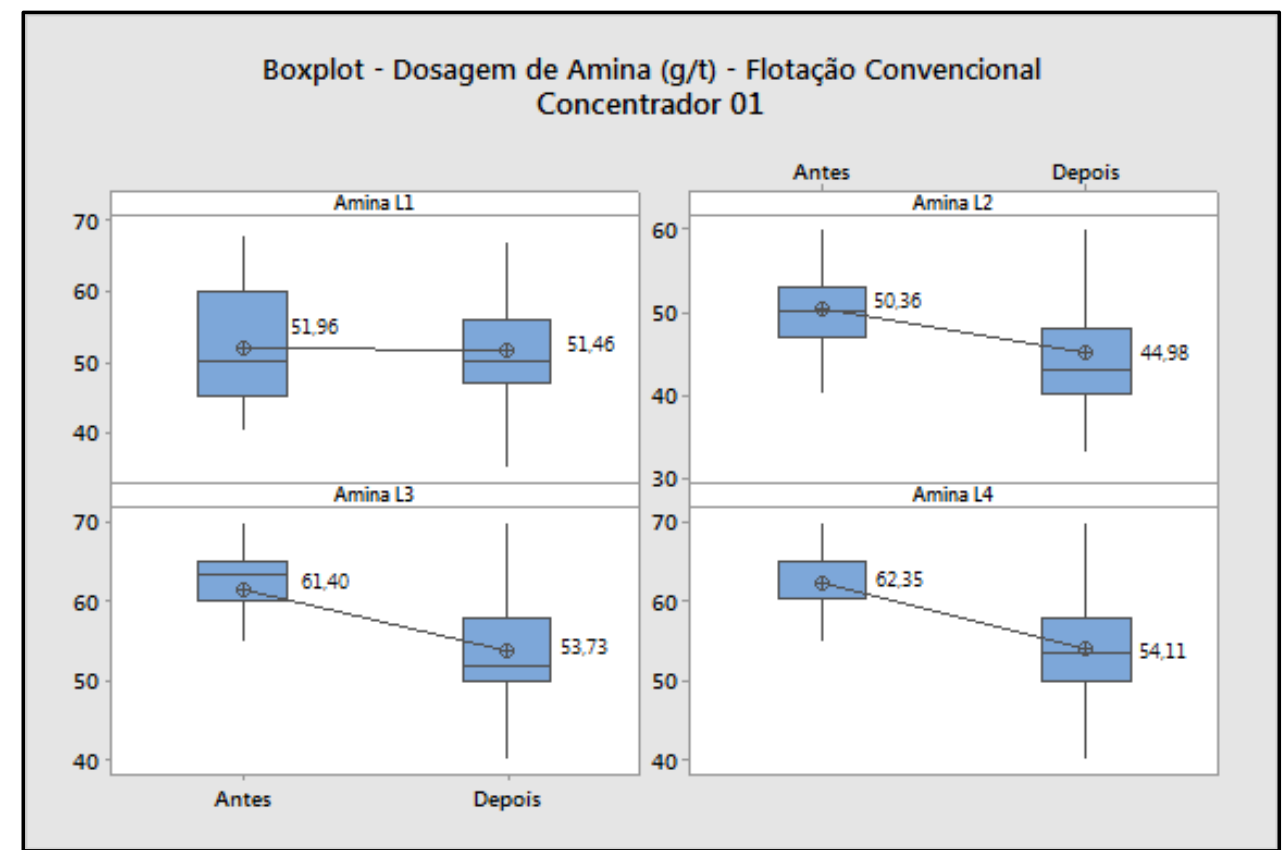

Figura 3: Redução na dosagem de amina (coletor) após a modificação do circuito.

Com as modificações o circuito tornou-se mais eficiente e os ganhos de produção são mostrados na tabela 5 .

Tabela 5 - Ganhos de produção após a modificação do circuito.

\begin{tabular}{|l|c|c|c|}
\hline \multicolumn{1}{|c|}{ Recuperações } & Circuito atual & Circuito modificado & Ganhos (\%) \\
\hline Recuperação em peso & 0,660 & 0,668 & $0,7078 \%$ \\
\hline Recuperação metálica & 0,92 & 0,93 & $1,07 \%$ \\
\hline \multicolumn{1}{|c|}{ Produção } & Circuito atual & Circuito modificado & Ganhos (t) \\
\hline t/h & 1539,47 & 1557,20 & 14,39 \\
\hline t/dia & 33621,96 & 34009,26 & 314,27 \\
\hline t/mês & 1008658,69 & 1020277,84 & 9428,24 \\
\hline t/ano & 12272014,05 & 12413380,35 & 114710,27 \\
\hline
\end{tabular}

Os ganhos anualizados de produção após a modificação do circuito são, aproximadamente, 114.700 toneladas e são medidos diariamente por meio de balanço de massas utilizando o software bilmat.

\section{CONCLUSÕES}

Conclui-se que o circuito modificado mantendo a etapa scavenger e transformando a etapa recleaner em cleaner da scavenger trouxe vários ganhos para o processo de flotação convencional como:

$\checkmark$ Redução do teor de ferro no rejeito global, com aumento da recuperação em massa e metalúrgica, o que reflete em um ganho de produção anual de 110.000t/ano de concentrado, na redução do custo específico e em ganhos ambientais; 
$\checkmark$ Redução do consumo de amina, uma vez que essa representa aproximadamente $22 \%$ do custo total de insumos utilizados no processo;

$\checkmark$ Redução da carga circulante proporcionando aumento do tempo de residência na flotação e ganho de seletividade, tornando o processo mais eficiente. Frequentemente, o alto percentual de carga circulante com baixo teor de ferro dificultava a obtenção de sílica e forçava o aumento da dosagem de reagentes para obtenção da qualidade. Esse aumento na dosagem gerava perdas de recuperação e aumento dos custos operacionais;

$\checkmark$ A disposição das células de flotação, após a modificação do circuito (concentrado final na etapa cleaner), permitiu que a alimentação dos moinhos secundários fosse feita por gravidade, o que motivou o fechamento do circuito dessa etapa de moagem e proporcionou ganhos de produtividade e redução do consumo energético.

\section{REFERÊNCIAS}

1 SANTANA,P. P (2012) - Flotação Direta de Minério de Ferro Itabirítico. Dissertação de Mestrado. Curso de Pós-Graduação em Engenharia Metalúrgica e de Minas). Escola de Engenharia da UFMG, Belo Horizonte.

2 COELHO, E. M. (1984) Beneficiamento de minérios de ferro. 1984. Tese (Concurso para professor titular)-Escola de Engenharia da UFMG, Belo Horizonte. Cap.3.

3 SILVA, D. B., VALADARES, M. C. (1988) Tratamento de minérios. Anais: Geologia, beneficiamento, caracterização e análise microestrutura de minérios de ferro. Vitória: ABM - Associação Brasileira de Metalurgia e Materiais.

4 VIANA, P. R. M. (2004) Prática industrial na indústria de minério de ferro. In: Apostila de Tratamento de Minérios. Departamento de Engenharia de Minas. Escola de Engenharia da UFMG, Belo Horizonte, p.205-225.

5 SANTIAGO, T. C., ARAUJO, A. C. et al (1993) Minério de ferro. In: Aspectos Tecnológicos Relativos à Preparação de Carga e Operação de Alto-Forno. ABM Associação Brasileira de Metalurgia e Materiais, Belo Horizonte. 